\title{
ERRATUM: “TIMING OF FIVE MILLISECOND PULSARS DISCOVERED IN THE PALFA SURVEY”
}

$$
\text { (2015, ApJ, 800, 123) }
$$

P. Scholz ${ }^{1}$, V. M. Kaspi ${ }^{1}$, A. G. Lyne ${ }^{2}$, B. W. Stappers ${ }^{2}$, S. Bogdanov ${ }^{3}$, J. M. Cordes ${ }^{4}$, F. Crawford ${ }^{5}$, R. D. Ferdman ${ }^{1}$, P. C. C. Freire $^{6}$, J. W. T. Hessels $s^{7,8}$, D. R. Lorimer ${ }^{9}$, I. H. Stairs ${ }^{10}$, B. Allen ${ }^{11,12,13}$, A. Brazier ${ }^{4,14}$, F. Camilo ${ }^{3}$, R. F. Cardoso ${ }^{9}$, S. Chatterjee ${ }^{4}$, J. S. Denneva ${ }^{15}$, F. A. Jenet ${ }^{16}$, C. Karako-Argaman ${ }^{1}$, B. Knispel ${ }^{12,13}$, P. LaZarus ${ }^{6}$,

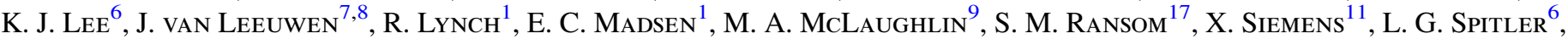

K. Stovall ${ }^{18}$, J. K. Swiggum ${ }^{9}$, A. Venkataraman ${ }^{19}$, and W. W. Zhu ${ }^{10}$

${ }^{1}$ Department of Physics, McGill University, Montreal, QC H3A 2T8, Canada; pscholz@physics.mcgill.ca

2 Jodrell Bank Centre for Astrophysics, School of Physics and Astronomy, University of Manchester, Manchester, M13 9PL, UK

${ }^{3}$ Columbia Astrophysics Laboratory, Columbia University, New York, NY 10027, USA

${ }^{4}$ Department of Astronomy, Cornell University, Ithaca, NY 14853, USA

${ }^{5}$ Department of Physics and Astronomy, Franklin and Marshall College, Lancaster, PA 17604-3003, USA

${ }^{6}$ Max-Planck-Institut für Radioastronomie, D-53121 Bonn, Germany

${ }^{7}$ ASTRON, Netherlands Institute for Radio Astronomy, Postbus 2, 7990 AA, Dwingeloo, The Netherlands

${ }^{8}$ Anton Pannekoek Institute for Astronomy, University of Amsterdam, Science Park 904, 1098 XH, Amsterdam, The Netherlands

${ }^{9}$ Department of Physics and Astronomy, West Virginia University, Morgantown, WV 26506, USA
${ }^{10}$ Department of Physics and Astronomy, University of British Columbia, 6224 Agricultural Road Vancouver, BC V6T 1Z1, Canada

${ }^{11}$ Physics Department, University of Wisconsin-Milwaukee, Milwaukee WI 53211, USA

${ }^{12}$ Leibniz Universität Hannover, D-30167 Hannover, Germany

${ }^{13}$ Max-Planck-Institut für Gravitationsphysik, D-30167 Hannover, Germany

${ }_{15}^{14}$ Cornell Center for Advanced Computing, Cornell University, Ithaca, NY 14853, USA

${ }^{15}$ Naval Research Laboratory, 4555 Overlook Ave SW, Washington, DC 20375, USA

${ }^{16}$ Center for Gravitational Wave Astronomy, University of Texas at Brownsville, TX 78520, USA ${ }^{17}$ NRAO, Charlottesville, VA 22903, USA

${ }^{18}$ Department of Physics and Astronomy, University of New Mexico, NM, 87131, USA

${ }^{19}$ Arecibo Observatory, HC3 Box 53995, Arecibo, PR 00612, USA

Received 2015 April 9; accepted 2015 April 21; published 2015 May 21

The names of the pulsars in our publication were based on the position of the PALFA survey beam in which each pulsar was discovered rather than their refined, pulsar-timing measured positions, as they should have been. Their names thus do not correspond to their actual right ascensions and declinations. Their correct names are: PSR J0557+1550, PSR J1850+0242, PSR J1901+0300, PSR J1904+0451, and PSR J1943+2210. We are grateful to Dick Manchester for pointing out this oversight to us. 